\title{
Role of Gastric Electrical Stimulation in the Treatment of Gastroparesis
}

\author{
Amal Shine and Thomas L. Abell * \\ Division of Gastroenterology, Hepatology and Nutrition, University of Louisville, 550 S. Jackson, ACB A3L15, \\ Louisville, KY 40202, USA; amal.shine@louisville.edu \\ * Correspondence: thomas.abell@louisville.edu; Tel.: +1-502-852-6991; Fax: +1-502-852-0846
}

Received: 31 October 2019; Accepted: 26 January 2020; Published: 31 January 2020

\begin{abstract}
Introduction: Gastric electrical stimulation (GES) is a surgically implanted treatment option for drug refractory gastroparesis syndromes. Evidence supporting use of GES and the pathophysiology of gastroparesis syndromes is not widely known. We conducted a descriptive review to elucidate the pathophysiology of gastroparesis syndromes, with particular focus on gastrointestinal neuromodulation and the known mechanisms of action of GES. Methods: A descriptive review of PubMed, Web of Science and Cochrane Library was conducted using the keywords gastric electrical stimulation, gastroparesis, nausea, vomiting, neuromodulation, gastroparesis syndromes, central nervous system, gastric pacing and electrical stimulation. Results: 1040 potentially relevant articles were identified, of which 34 were included. These studies explored various central and peripheral effects of GES, as well as its effect on quality of life, hospital stay, mortality and health-related costs. Conclusion: Although evidence supporting gastrointestinal (GI) electrical stimulation and GI neuromodulation use is not widely known, GES does seem to offer significant improvement in symptom control, quality of life and other effects to many patients. GES exerts its effects through multiple central and peripheral mechanisms and has potential to modify the natural history of disease. Future work on gastroparetic syndromes and their treatment might be better focused in terms of pathophysiologic mechanisms. Improving outcomes with specific neuromodulation therapies, like GES, may offer improvements in health for many patients with refractory upper gastrointestinal symptoms.
\end{abstract}

Keywords: gastric electrical stimulation; gastroparesis; nausea; vomiting; neuromodulation; gastroparesis syndromes; central nervous system; gastric pacing and electrical stimulation

\section{Introduction}

Gastroparesis is traditionally defined as a syndrome of objectively delayed gastric emptying in the absence of mechanical obstruction and cardinal symptoms. Symptoms of gastroparesis include nausea, vomiting, bloating, early satiety and abdominal pain. While the exact incidence of gastroparesis is not well-established, this disorder was reported as a primary cause of hospitalization in about 10,000 patients and as a secondary diagnosis in another 134,000 admissions, between 1995 to 2004 [1]. Gastroparesis can be severely debilitating, and treatment is often difficult. High-frequency, low-energy gastric electrical stimulation (GES) has been reported to reduce symptoms of drug-refractory gastroparesis [2]. The United States Food and Drug Administration (FDA) approved the use of GES to treat drug-refractory idiopathic and diabetic gastroparesis in the year 2000. This FDA approval, for the current Enterra system (Medtronic, Inc.), allows for use of higher than nominal energies of stimulation. In addition, a trial of temporary gastric stimulation may help determine patients who would benefit from surgical placement of a permanent gastric stimulator [3]. This article reviews some of the new findings on the pathophysiology of gastroparesis syndromes and mechanisms of action of 
GES, while focusing on one particular therapy—GI neuromodulation via low and medium energy gastric electrical stimulation (GES).

\section{Background}

Gastroparesis is well-described in patients with delayed gastric emptying. An emerging concept, proven by increasing evidence and demonstrated by pathophysiology, is gastroparesis-like syndrome (GLS), otherwise known as unexplained nausea and vomiting (UNAV), besides classic gastroparesis (Gp). The addition of GLS to Gp, which together can be termed as gastroparesis syndromes, helps include patients who exhibit the symptoms of gastroparesis but have a normal or even rapid emptying study. It is important to note that Gp and GLS/UNAV do not comprise patients with cyclic vomiting syndrome, or those who present with only nausea and vomiting and none of the other cardinal symptoms that indicate a gastric origin. Such patients require investigation to rule out non-gastrointestinal causes such as vestibular or central nervous system dysfunction, cannabis use or medications. To describe therapies such as neuromodulation for the symptoms of Gp or GLS/UNAV, it is important to review what is known about the pathophysiology of both of these disorders, which is now better understood by the changes demonstrated after low and medium energy gastric electric stimulation. A recent study demonstrated low and medium energy GES effects in five major areas: inflammatory, autonomic, enteric, electrophysiologic and hormonal [4]. Additional studies have also explored the role of the central nervous system in gastroparesis. These mechanisms, elaborated below, seem to affect and perhaps alter important elements that allow the normal gastric motor function, such as changes in nerves, muscle cells, and interstitial cells of Cajal (ICC).

\section{Treatment of the Symptoms of Gastroparesis-An Overview}

The symptoms of gastroparesis can be treated with a variety of modalities. A recent summary, written for patients, classified the treatments of Gp syndromes into five categories [5]: 1. Diet: Diet modification is a reasonable initial approach. 2. Drugs: Drugs, or pharmacotherapy, typically with metoclopramide or erythromycin and newer prokinetics are the next line of treatment. Unfortunately, around $30 \%$ of patients fail conservative management [6]. 3. Devices: If patients are refractory to drugs, devices such as GES, especially as a temporary endoscopy trial, could be considered. 4. Direction: Newer approaches such as 'diverting' or disrupting the pyloric sphincter endoscopically or surgically using pyloromyotomy are also being used, especially if there is pyloric dysfunction and delayed emptying. A recent study of 34 patients also demonstrated that pyloric injections of botulinum toxin $\mathrm{A}$ provided symptomatic improvement at 1 month in $64 \%$ of patients, with improvement often extending up to 6 months [7]. 5. Detoxify: Many patients, particularly those with neuromuscular issues from systemic inflammation, benefit from intravenous immunoglobulin, or other immune modulators, which "detoxifies" the immune system. This article will focus on one of these categories: devices, particularly neuromodulation devices.

\section{Neuromodulation as a Bio-Electric Therapy for Gastroparesis Symptoms}

A number of devices have been used for treatment of Gp syndromes as neuromodulators via bio-electronic means. These devices include thalamic, auricular, electro-acupuncture, vagal, spinal and enteric devices, such as GES, using low, intermediate or high energies. The therapeutic potential and avenues for use of GES are extensive, with promising implications for the future of GI motility disorders. GES exerts its effect through multiple mechanisms in patients with gastroparesis symptoms. Through literature review and compilation of findings from prior studies, we discuss below the various clinical effects of GES: symptom improvement, central effects, anti-emesis, pro-kinetic, anti-arrhythmic, anti-inflammatory, hormonal stabilization, autonomic action, improved pancreatic function, mid-gut and hind-gut effect, improvement in glycated hemoglobin, improved quality of life, as well as decreased hospital costs. While not an exhaustive list, these mechanisms are the best studied and reported so far. 


\section{Demographics of GES}

Gastric electrical stimulation is used in both the United States and Europe, but only limited data exist about the demographics of gastroparesis patients receiving GES by geographic location. A recent study by Burlen et al. [8] addressed this issue and also reviewed the current evidence-based data on GES. This US-European study demonstrated significant differences in gastroparesis demographics and percentage of patients, starting with gastric emptying test (GET) data. European centers had 61 GES patients compared to 319 from the US. In Europe, 100\% of patients had GET values available in the published data; in the US, it was $75 \%$ of patients. European centers had more diabetes patients $(59 \%)$ than the US (22\%), and a smaller proportion of idiopathic Gp patients $(25 \%)$ than the US $(72 \%)$, with a statistical difference between the causes of gastroparesis in the patients receiving GES between the two geographical areas $(p<0.00001)$. There was also significant difference in the gender of the patients receiving GES, with a greater proportion of women in the US $(p=0.0023)$. This US-European comparison emphasizes the need for a widely available, easily accessible comprehensive database that allows better analysis and treatment of gastroparesis patients worldwide, particularly regarding neuromodulation therapies.

\section{Details on Mechanism of Action of GI Neuromodulation}

The following sections discuss the impact of neuromodulation on the GI tract. It is important to mention that although most patients with GES start out at nominal/truly low energy settings, many require a higher than nominal energy setting for optimal control of symptoms, and those energies can be better classified as intermediate/moderate energy GES [9].

\subsection{Central Effect}

Most early studies were focused on the peripheral effects of GES. Hence, little was known about GES utilization of and effects on central mechanisms. In animal models, involvement of the vagal afferent pathway was studied using various methods of gastric stimulation. GES was found to activate neurons responsive to gastric distension in the nucleus tractus solitarii, confirming the involvement of the vagal afferent pathway [10].

A 2019 case-control study by Snodgrass et al. [11] used functional MRI to investigate the central nervous system mechanisms of nausea in patients with gastroparesis. This study used a 30-min visual stimulation using a flashing/rotating checkerboard image on a computer screen to induce nausea. Results revealed decreased bilateral insular connectivity compared to the right insula and temporal lobe, in study patients versus healthy controls (Figure 1). This phenomenon may not only explain increased susceptibility of patients with gastroparesis to nausea but also causation by chronic nausea.

Data suggest that the symptomatic improvement achieved by GES in gastroparesis is explained by multiple processes. The activation of vagal afferent pathways by GES appears to influence CNS control mechanisms for nausea and vomiting. Enhanced vagal efferent autonomic function and decreased gastric sensitivity to volume distention also enhances postprandial gastric accommodation with GES [12]. This understanding may lead to more therapeutic options or devices aimed at vagal autonomic neuronal modulation, in order to improve symptoms of gastroparesis. However, other mechanisms of varying significance remain to be studied for causation. 


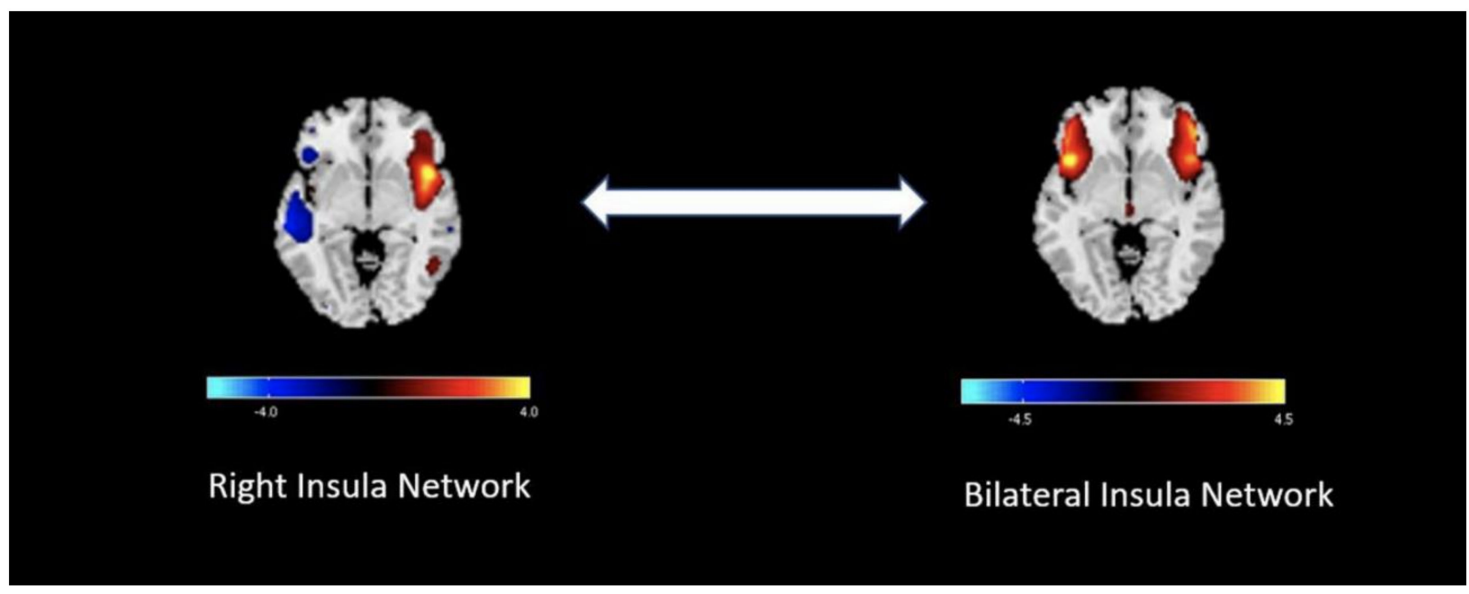

Figure 1. Central effect. The fMRI brain images (see bidirectional arrow) depicting a component containing maximal values in the right insula extending into left temporal lobe described as right insula network and a component containing right and left insula described as bilateral insula network that were compared after exposure to a nausea-inducing visual stimulus in 10 gastroparetics and 8 healthy controls. The functional network connectivity was significantly reduced $(p<0.001)$ in gastroparetic patients versus healthy controls after visual stimulation between the right insula network and bilateral insula network. The highlighted areas illustrate the locations of reduced connectivity in gastroparesis [11].

\subsection{Anti-Emetic Effect}

Intractable nausea and vomiting are common symptoms of gastroparesis. Multiple studies have demonstrated the effect of GES on emesis. In a 2006 study of eleven patients, with both idiopathic and diabetic gastroparesis, GES at a frequency of 12 cycles/minute had an immediate anti-emetic effect, in as early as one week [13]. Other data evidence that $80 \%$ of patients have at least $50 \%$ symptom improvement with GES, and $50 \%$ of patients have approximately $80 \%$ improvement in symptoms $[14,15]$.

In 2011, a double-masked randomized cross-over study reported decreased vomiting scores as well as total symptoms scores in patients, when temporary mucosal endoscopic GES was activated, compared to when it was not [16]. In the first session, vomiting decreased with and without stimulation, but was greater with stimulation, resulting in a day 3 difference of $-1.02(p=0.001)$. However, GI symptom scores did not return to baseline during washout; on day 4 , the difference persisted at $-1.08(p=0.005)$. In the second session, vomiting slightly decreased with stimulation and slightly increased without it; at day 8 , the group without stimulation had non-significantly greater vomiting, $0.12(p=0.762)$. Of note, the two groups, although randomized, were not equal in symptoms or baseline physiology measures, which may have influenced the outcomes of the study. Thus, this randomized, cross-over study showed that when endoscopically placed, temporary GES (tGES) may reduce symptoms, such as nausea and vomiting.

Another recent study examined effects of GES in an open-label manner and found nausea and vomiting were significantly improved from baseline after both temporary and permanent GES at 5-7 days and 6 months, respectively. When measured using the Gastroparesis Cardinal Symptom Index (GCSI) scale, nausea was demonstrated to have reduced from a baseline level of 3.5 to 1.7 and 2.6, with the placement of a temporary and permanent GES. Similarly, GCSI vomiting scores reduced from the baseline of 2.4 to 0.6 and 1.8 [4] (Supplementary Table S1). Thus, primarily designed to explore mechanisms of GES, using both low and medium energy, this study supports the use of GES to combat nausea and vomiting in patients. 


\subsection{Prokinetic Effect}

Several studies have shown the improvement of gastric emptying after placement of GES. A previous study (2003) demonstrated significant improvements to gut motility resulting from low-energy GES placement in both patients with diabetic and idiopathic gastroparesis. Two- and four-hour gastric emptying improved significantly at 6 and 12 months for the combined group, with two-hour retention reducing from $78 \%$ to $56 \%(p<0.05)$, at twelve months. Diabetic patients also experienced significant decline in gastric retention at 4 hours, decreasing from $46 \%$ to $16 \%(p<0.05)$, in twelve months of use [14]. The mechanism by which GES increases gastric emptying could be more explained by increased gastric accommodation rather than increased propulsive gastric waves. Gourcerol et al. showed that GES increased gastric maximal tolerable volume (MTV) to distension from $522 \pm 64 \mathrm{~mL}$ at baseline to $628 \pm 60 \mathrm{ml} 6$ months after the start of GES ( $p=0.03$ ) [17].

In a more recent study, the prokinetic effect measured by gastric emptying in patients with delayed baseline emptying was found to start early and be sustained. In patients with any baseline gastric emptying delay, total liquid emptying significantly decreased, from $94 \%$ at baseline to $52 \%$ and $58 \%$, with the use of a temporary and permanent GES, respectively. Additionally, baseline total solid emptying decreased from $152 \%$ to $105 \%$ and $100 \%$ at 6 days and 6 months [4] (Figure 2). In this study, which used moderate energy GES, accelerated gastric emptying of patients with delayed baseline gastric emptying may aid in abatement of symptoms and complications, arising secondary to gastroparesis.
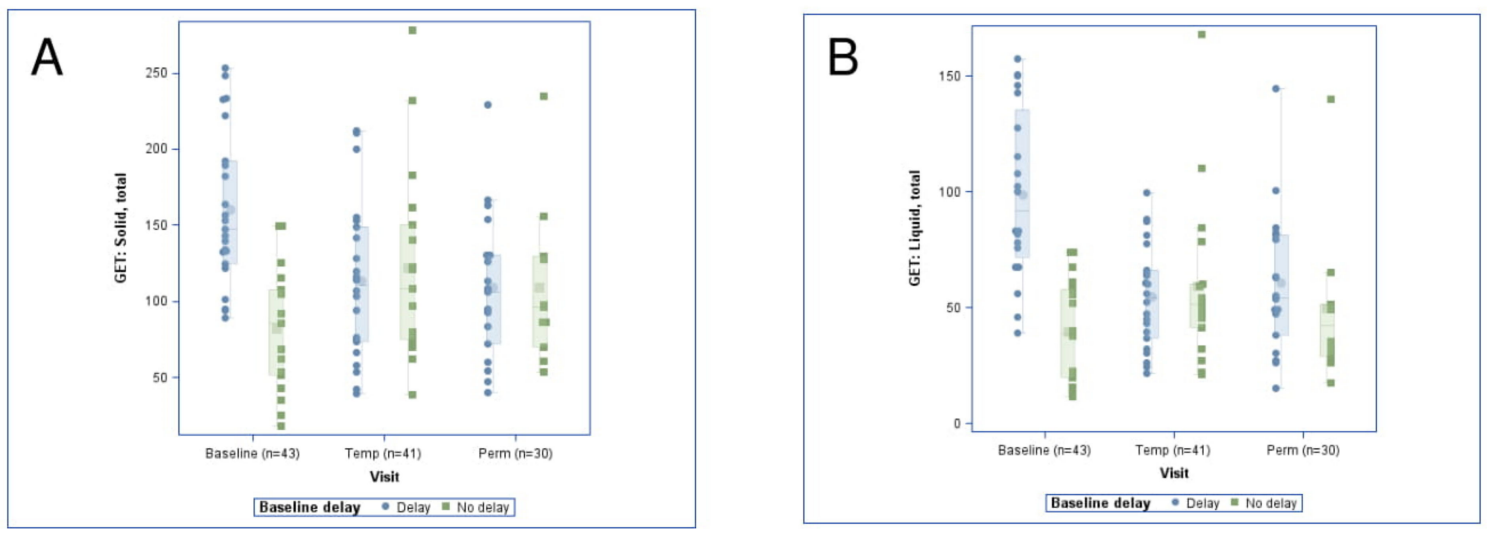

Figure 2. Pro-kinetic effect with low and medium energy gastric electrical stimulation (GES). Stratified changes over the study period in solid (A) and liquid (B) gastric emptying according to delay status based on either 1-h liquid or 4-h solid retention [4].

\subsection{Antiarrhythmic Effect}

The antiarrhythmic effect of GES has been shown in recent studies which have used both mucosal (mEG) and cutaneous electrogastrograms (EGG). Cutaneous EGG is a non-invasive technique to record gastric myoelectrical activity via electrodes placed on the abdominal surface, while mucosal EG is a non-operative procedure requiring endoscopy to measure gastric electric waves using mucosally placed electrodes. Both of these measures (EGG and $\mathrm{mEG}$ ) can be done as low- or high-resolution techniques. Frequency, amplitude, and frequency-amplitude-ratio of cutaneous EGG and mucosal (and likely, serosal) electrograms have been shown to be reliable measures of gastric motility [18].

In 2019, the previously mentioned National Institutes of Health (NIH) study [4] revealed an early and significant antiarrhythmic effect, seen in cutaneous EGG frequencies in all patients (baseline: 5.3 cycles per minute (CPM), temporary (day 6) GES: 4.6 [Padj = 0.003], permanent (month 6) GES: 4.4) and in delayed gastric emptying patients (baseline: 5.6 CPM, temporary GES: 4.7 [Padj $=0.02$ ], permanent GES: 4.4). Similar changes were seen with mucosal electrogram (mEG) frequency for diabetic patients (baseline: 4.9 CPM, temporary GES: 3.8, Padj = 0.02), but not for patients with idiopathic gastroparesis (baseline: 4.6, temporary GES 4.1, Padj = 0.69) (Supplementary Table S2). Since loss of normal gastric 
electrical rhythm may be contributory to gastroparesis, this targeted antiarrhythmic action of GES may help restore baseline motility.

\subsection{Anti-Inflammatory Effect}

Emerging evidence suggests that patients with gastroparesis may have systemic inflammation, with elevation of serum pro-inflammatory markers. In a 2011 study, a patient group with symptoms of gastroparesis were shown to have increased inflammatory cytokines at baseline [19]. In a more recent study [4], changes in cytokine levels began after use of a temporary GES, and there was a marked decrease in inflammatory markers at 6 months after permanent GES. Patients with gastroparesis symptoms had elevated levels of interleukin-6 (IL-6) $(46.64 \pm 53.01 \mathrm{pg} / \mathrm{mL})$ and tumor necrosis factor alpha $(\mathrm{TNF} \alpha)(22.18 \pm 7.46 \mathrm{pg} / \mathrm{mL})$ at baseline (normal IL- $6<10.1 \mathrm{pg} / \mathrm{mL}$ and TNF $\alpha<7.1 \mathrm{pg} / \mathrm{mL}$ ). Following the placement of temporary GES, IL-6 levels increased (141.74 $\pm 133.04 \mathrm{pg} / \mathrm{mL})$, while TNF $\alpha$ levels began to decrease $(19.84 \pm 8.50 \mathrm{pg} / \mathrm{mL})$ after 6 days. At 6 months with permanent GES, IL-6 $(15.34 \pm 20.51 \mathrm{pg} / \mathrm{mL})$ and TNF $\alpha$ levels $(6.58 \pm 2.58 \mathrm{pg} / \mathrm{mL})$ were found to have diminished to near normal ranges (Padj < 0.001) (Figure 3).
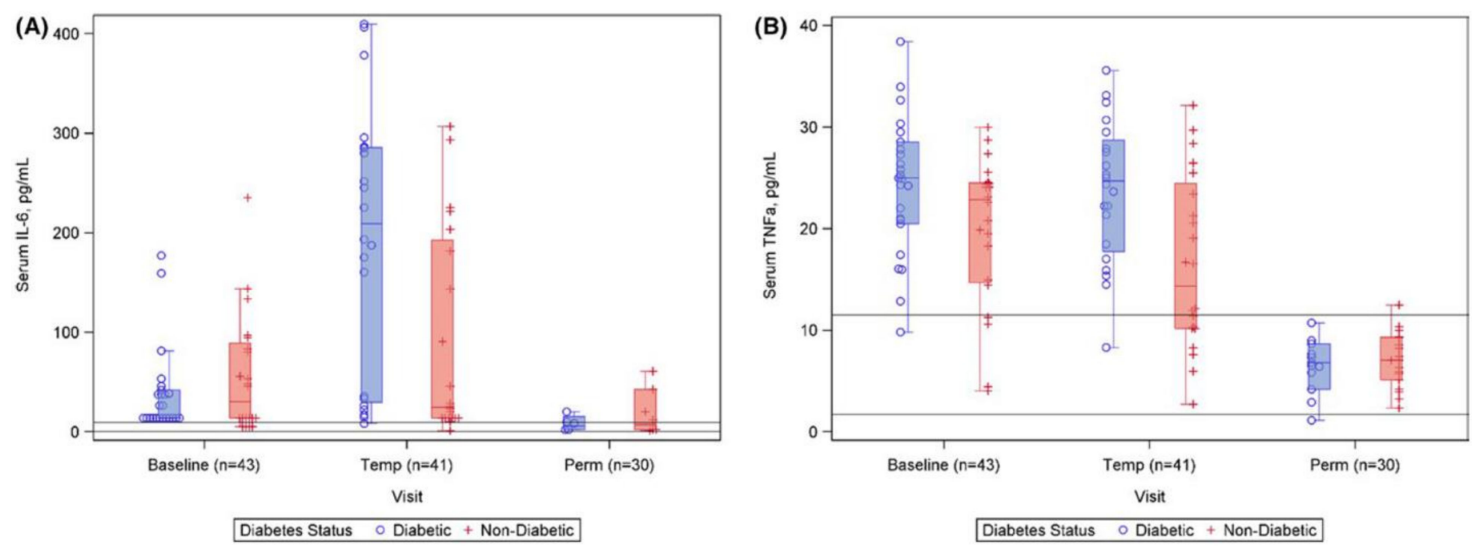

Figure 3. Anti-inflammatory effect. Inflammation changes during the NIH DiaComp study: baseline, 6 days and 6 months using low and moderate energy GES, stratified by diabetes status, measured by IL-6 $(\mathrm{pg} / \mathrm{mL})(\mathbf{A})$ and TNF- $\alpha(\mathbf{B})[4]$.

\subsection{Hormonal Stabilization Effect}

Previously, animal studies have demonstrated hormonal effects of GES, often with high energy, as alteration in ghrelin and cholecystokinin levels [20,21]. In the more recent DiaComp NIH study [4] conducted in patients with gastroparesis symptoms, appetite hormones (leptin and ghrelin) did not significantly change with GES. However, late effects were observed in metabolic hormones (insulin, glucagon, amylin; Padj $<0.001$ for all) in all patients. Following temporary GES, diabetic patients had significantly higher serum insulin $(9373 \mathrm{pmol} / \mathrm{L}$ vs. $3652 \mathrm{pmol} / \mathrm{L}, \mathrm{Padj}=0.03)$ and serum glucagon $(276 \mathrm{pg} / \mathrm{mL}$ vs. $189 \mathrm{pg} / \mathrm{mL}$, Padj $=0.03)$ compared to those suffering from idiopathic gastroparesis (Figure 4). Of note, many of these patients had abnormal hormone levels at baseline. Due to its influence on endocrine and exocrine systems, GES may find application in targeted treatment of causal mechanisms of gastroparesis, whether diabetic or not. 

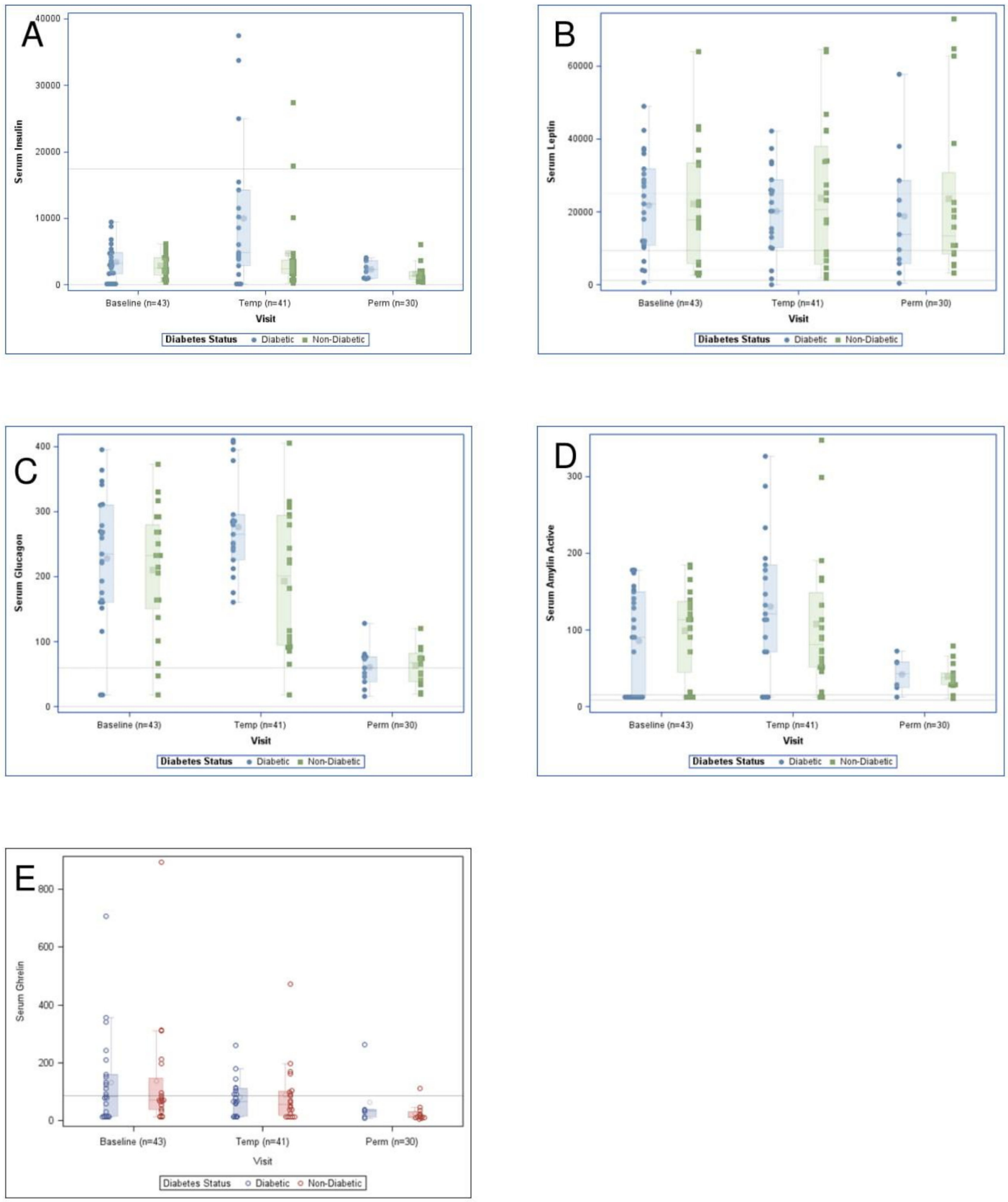

Figure 4. Hormonal stabilization effect. Stratified changes over the observational period (baseline, 6 days and 6 months) of serum levels of insulin (A), leptin (B), glucagon (C), amylin (D), and ghrelin (E) in response to low and medium energy GES [4].

\subsection{Improved Quality of Life}

Several studies have demonstrated an improvement in quality of life (QOL) with the use of GES, as measured by Short Form 36 (SF-36) or Investigator Derived Outcome Measurement System (IDIOMS), which is a Medicare DRG adapted tool. In a long-term follow-up of 28 patient with GES that focused on nutritional aspects of Gp syndromes, a significant postoperative increase of $40.7 \%$ and $24.6 \%(p<0.05)$ in quality of life scores was demonstrated [22] (Supplementary Table S3).

In a more recent study, early and persistent improvements in health-related quality of life were seen with GES, as measured by composite illness scores using IDIOMS, $(p<0.001)$ for all baseline to permanent comparisons. Changes in total IDIOMS scores with GES were more pronounced in 
idiopathic patients (baseline:19.9, temp GES:12.8, perm GES:13.5) compared to diabetic patients. (baseline: 19.9, temp GES:13.3, perm GES:14.4) [4].

\subsection{Autonomic Effect}

Gastroparesis is often a manifestation of gastrointestinal, neuromuscular and/or autonomic dysfunction. Many patients with drug refractory Gp syndrome with autonomic abnormalities respond to neuromodulation via GES, which also appears to influence the autonomic nervous system. Both traditional direct autonomic measures and indirect measures such as heart rate variability have been evaluated to study these effects. Stocker et al. conducted a study in 2016 [23] to evaluate changes in autonomic measures after GES. Cholinergic function was evaluated using two methods: changes in the R-to- $R$ interval in response to three cycles of deep respiration; and the Valsalva ratio, represented by the change in heart rate in response to forced respiration for $15 \mathrm{~s}$ with a constant pressure of $40 \mathrm{~mm}$ $\mathrm{Hg}$. Both the R-to-R interval and the Valsalva maneuver were summed and reported as cardio-vagal cholinergic function. Adrenergic function was assessed using an infrared photoplethysmography attached to the patient's index or middle finger. Capillary vasoconstriction reflexes in response to cold immersion and postural changes were recorded. Values from the adrenergic tests were added together and reported as sympathetic function. Both systemic autonomic testing and heart rate variability measures were frequently abnormal at baseline and showed changes after GES therapy, in two groups of symptomatic patients (Figure 5).

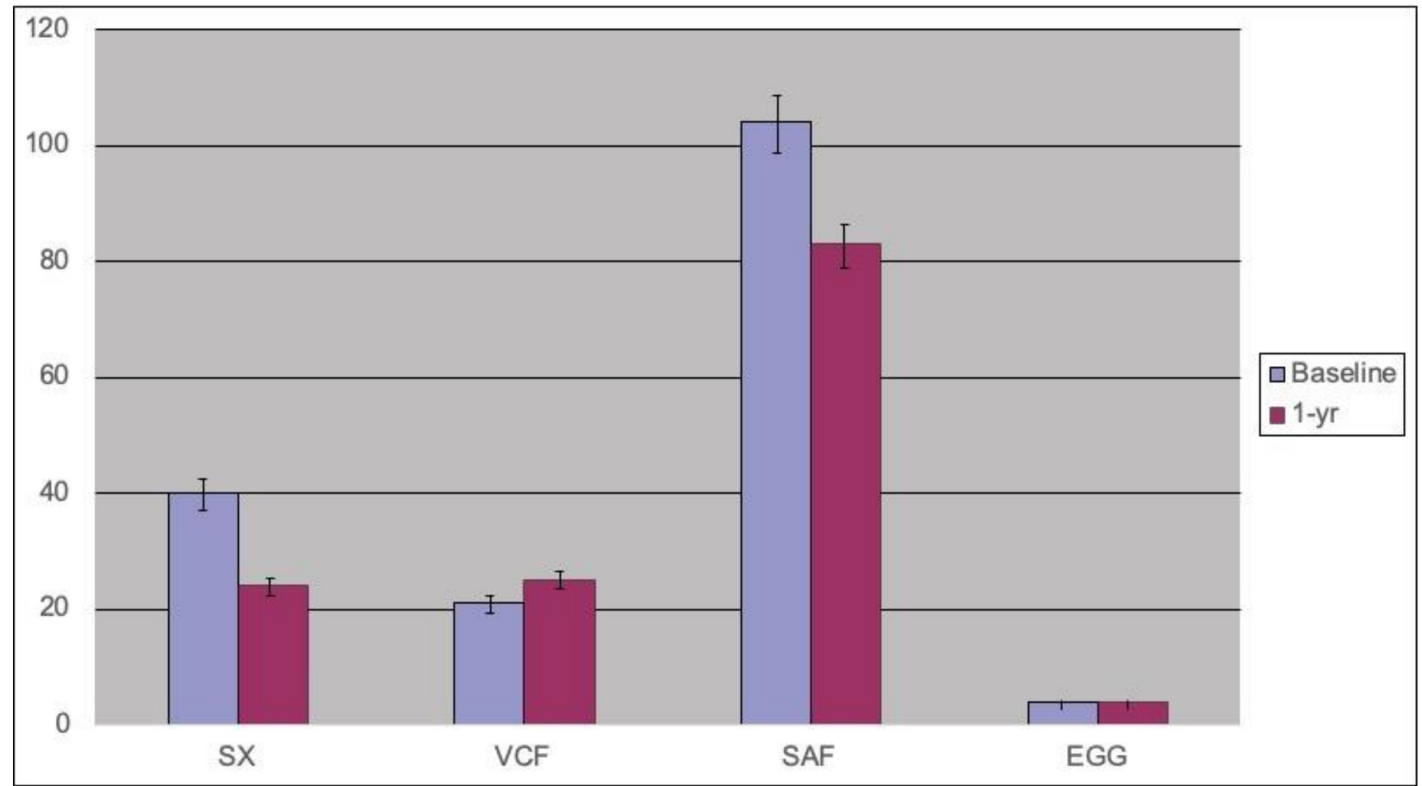

Figure 5. Autonomic effect. Symptoms, autonomic and enteric measures at baseline and 1 year after gastric stimulation. Legends: SX: Symptoms; VCF: vagal cholinergic function; SAF: sympathetic adrenergic function; EGG: electrogastrography [23]. Upper GI symptoms (Sx) are scored on 0-10 scale for 5 common Gp Sx. Vagal cholinergic function (VCF) and sympathetic adrenergic function (SAF) are measured in standardized units. Electrogastrogram (EGG) frequency is reported in cycles per minute.

\subsection{Improved Pancreatic Function}

There is some evidence suggesting a correlation between chronic pancreatitis, especially small-duct chronic pancreatitis, and gastroparesis [24]. Luo et al. [25] demonstrated that GES is associated with improvement in human pancreatic exocrine function in a randomized, blinded study. Fecal elastase values were compared in two patient groups: (1) GES devices ON and (2) GES devices OFF and (3) in control groups. Elastase level was different for GES-ON and OFF (508.0 $\pm 92.2 \mu \mathrm{g} \mathrm{E1/g} \mathrm{stool}$ vs. GES-OFF $378.6 \pm 87.4 \mu \mathrm{g}$ E1/g stool, $p<0.05$ ). It was also noted that elastase levels were lower 
in medication non-responders and responders, versus normal controls. Postprandial pancreatic polypeptide levels were greater with GES ON than OFF $(p=0.08)$ (Figure 6$)$. This suggests a possible role for GES in the treatment of pancreatic insufficiency associated with gastroparesis.

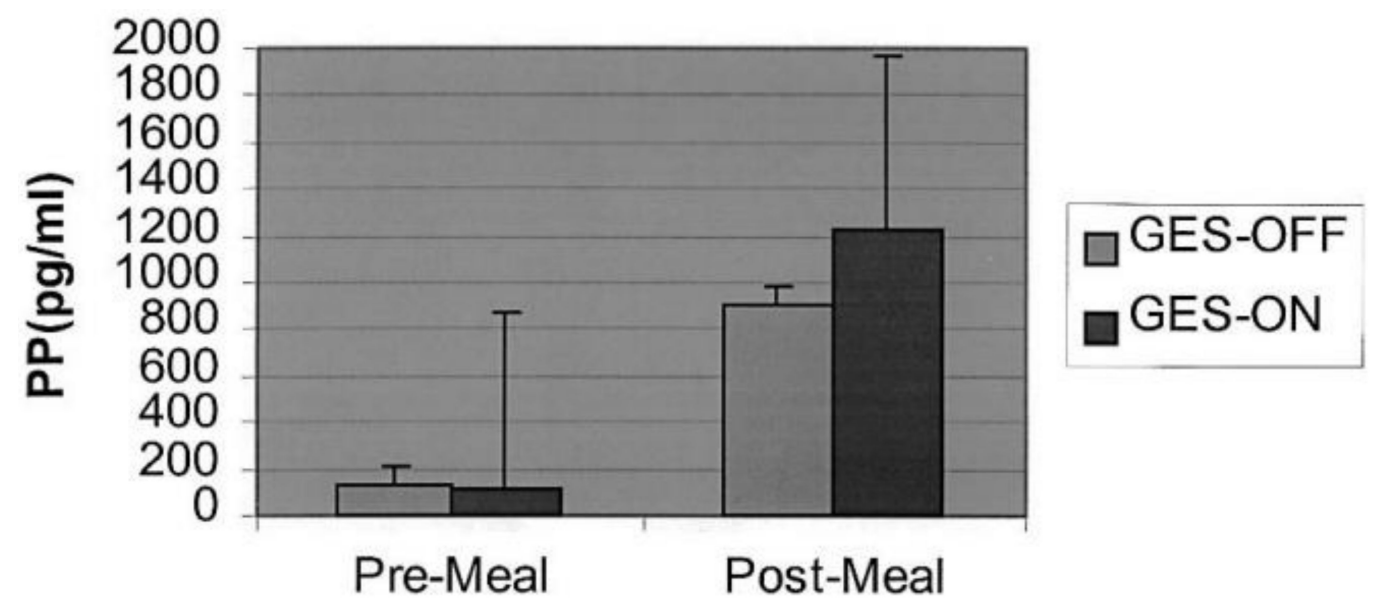

Figure 6. Improvement in pancreatic function in a controlled, randomized and blinded study of GES. Serum pancreatic polypeptide (PP) level in 7 patients with gastroparesis in relation to meal and gastric electrical stimulation (GES-OFF and GES-ON). PP was similar at lowest baseline level between GES-OFF and GES-ON $(p>0.3)$. Highest PP level after the meal was greater with GES-ON than GES-OFF $(p=0.08)$ [25].

\subsection{Decrease in Hospital Days}

Patients with gastroparesis experience frequent and prolonged hospitalizations owing to the inability of traditional treatment methods to control their nausea, vomiting and pain. A controlled, multicenter, prospective study was published in 2010 and evaluated the safety and efficacy of GES in patients with chronic intractable nausea and vomiting from diabetic gastroparesis. Amongst other conclusions, this study demonstrated a significant decrease in hospital days after GES, demonstrating a reduction in annualized median days in the hospital, from 2 days at baseline to 0 days at the end of one year $(p=0.006)$ [26]. Fewer hospitalizations and shorter duration of stays in turn would lead to fewer co-morbidities, costs and hospital-acquired diseases.

\subsection{Relationship to Cajal Cell Counts}

Interstitial cells of Cajal (ICC) are mesenchymal and neural crest derived cells that occur throughout the muscular layers of the gastrointestinal tract and play an important role in the generation and propagation of electrical slow waves. They also mediate bidirectional communication between the autonomic nervous system and smooth muscle cells of the gastrointestinal tract. An animal study by $\mathrm{Li}$ et al. examined changes in ICC utilized GES with different energies as well as a sham stimulator. The distribution of ICCs was observed using immunolabeling for c-kit, while smooth muscle cells were identified using $\alpha$-SMA antibodies. Improvement in ICC and smooth muscle cells was observed in all GES groups, especially in the low frequency-high energy group [27].

In a recent study, electrograms obtained after GES demonstrated immediate improvement in gastric electrical activity and gastroparesis symptoms in patients with relatively higher ICC counts, compared to patients with extensive loss of ICC [28] (Supplementary Table S4). This suggests that GES may have a role in potentiating the function of ICC.

\subsection{Decrease in Health-Related Costs}

The number of inpatient admissions for gastroparesis and associated costs have increased significantly in recent years. Cutts et al. (2005) studied patients with symptoms of gastroparesis over three years and demonstrated that GES, as compared with intensive medical therapy, is more effective 
in improving long-term gastrointestinal symptoms and costs, while decreasing use of healthcare resources, compared to intensive medical therapy [29]. Gastrointestinal symptoms were significantly different from baseline ( $F=3.03, p<0.017)$ in patients treated with GES, showing more sustained improvement over 36 months than those treated with medical therapy. Healthcare resource usage, measured in part by the Medicare derived tool of IDIOMS, significantly improved at 12-, 24- and 36-month follow-ups for patients on GES therapy $(F=10.49, p<0.001)$, compared to patients receiving medical therapy, who demonstrated further deterioration. GES also proved superior to medical therapy at 24 and 36 months with regard to decreased costs $(F=4.85, p<0.001)$ (Figure 7). Within-group comparisons indicated significantly reduced hospital days for both GES and intensive outpatient medical therapy patient groups.

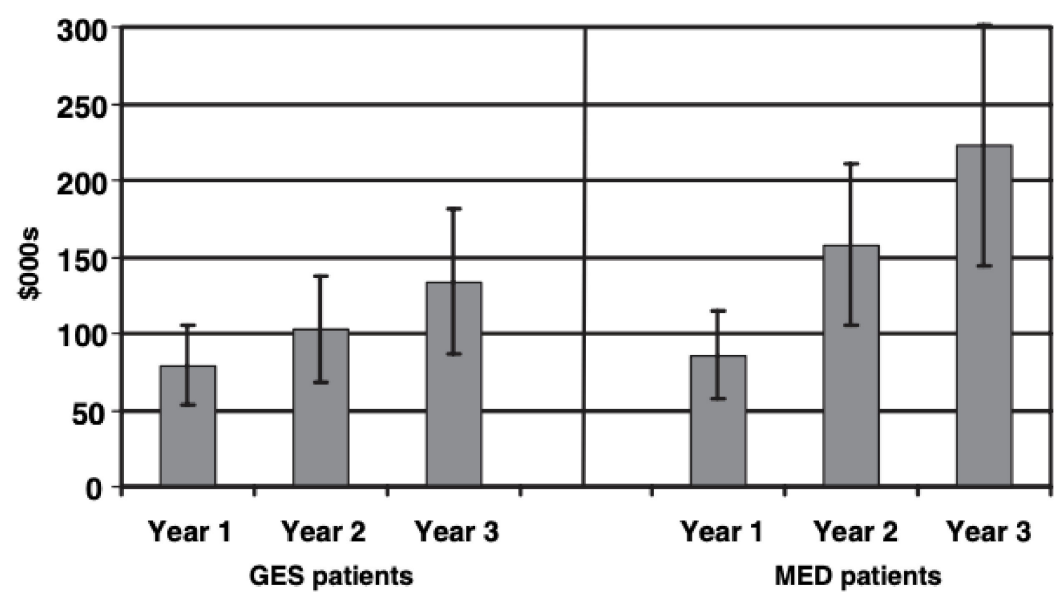

Figure 7. Decrease in health-related costs with GES vs. medical controls. Comparison of health-related costs of GES patients and patients treated with outpatient medical therapy in a long-term study. Cumulative costs for the first, second and third years after enrolment in the study [29].

\subsection{Effect on Pain}

Pain can be a debilitating symptom of gastroparesis and is often the reason for many hospital admissions. Prior studies have shown that GES placement can help reduce nausea and total symptoms scores. A study published in 2013 [30] focused on the effect of GES on abdominal pain. Ninety-five Gp patients' symptoms were recorded at baseline and during temporary and permanent GES. Sixty-eight patients reported severe pain at baseline. Severe pain patients' mean pain scores decreased with temporary GES from 3.62 to $1.29(p<0.001)$ and non-severe pain from 1.26 to $0.67(p=0.01)$. With permanent GES, severe mean pain scores fell to $2.30(p<0.001)$; non-severe pain changed to 1.60 $(p=0.221)$. Mean follow-up was 275 days (Supplementary Table S5). Patients with uncontrolled pain may be good candidates for GES therapy.

\subsection{Mid-Gut and Hind-Gut Effect}

Newer studies have elucidated the effects of GES on the mid gut and hind gut. In a 2016 study [31], 54 patients who underwent dual-device treatment (GES and sacral electrical stimulation) were followed for 24 months. With combined treatment, a statistically significant improvement was seen in upper GI, lower GI and genitourinary symptoms as well as quality of life.

In a more recent combined animal and human study [32], the effects on the mid-gut were also explored. In the animal model, high-resolution electrical slow wave mapping of frequency, amplitude, and their ratio, for duodenal and Sphincter of Oddi electrical activity were recorded. In the human study, eight patients underwent temporary gastric stimulation with small bowel electrodes. Symptom scores, gastric emptying times, and mucosal electrograms via low-resolution mapping were recorded before and after gastric stimulation for the patients. Gastric electrical stimulation in the animal model showed non-significant effects on small bowel slow-wave activity and myoelectric signaling, suggesting 
the existence of intrinsic neural connections (Figure 8). Human data showed more significance, with possible potential for therapeutic use of electrical stimulation in patients with gastroparesis and pancreato-biliary and small bowel symptoms of the mid-gut. These animal and human studies show the probable effects of GES on other sites of the gut apart from the stomach.

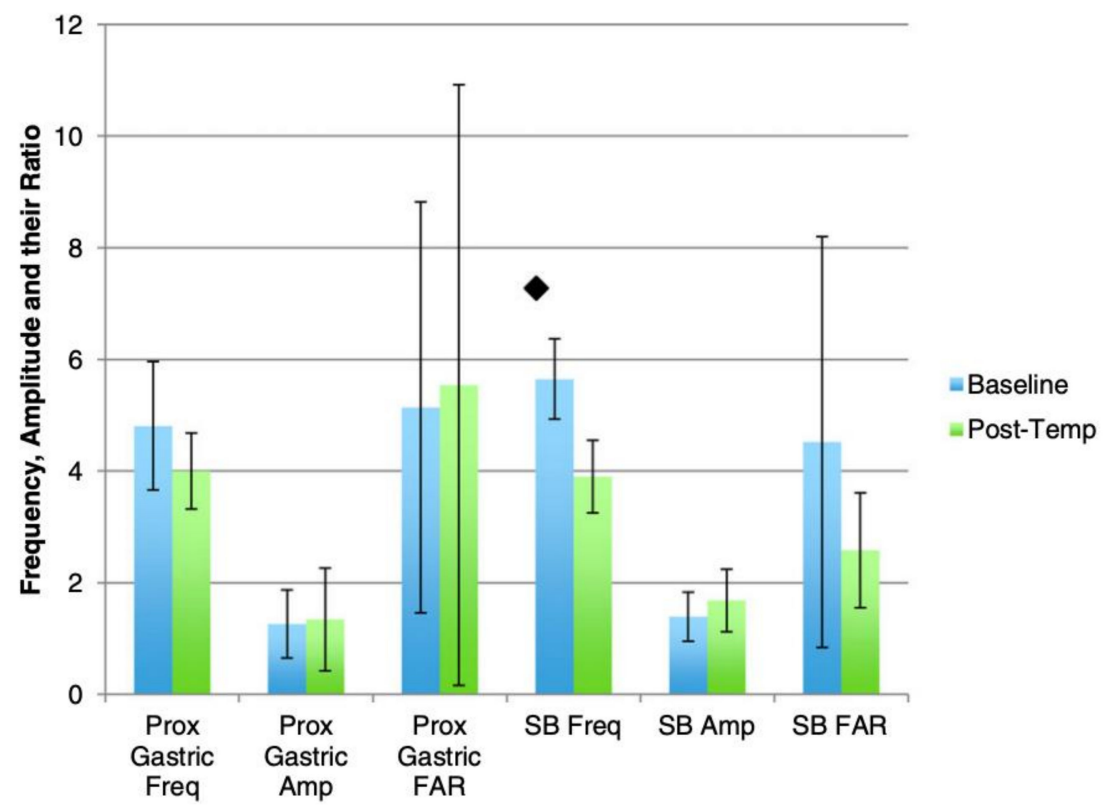

Figure 8. Midgut effects of medium-energy temporary endoscopic mucosal GES on gastric and small bowel electrical slow wave activity [32]. Frequency of the mucosal electrograms was in cycles per minute, amplitude in milliamps, and their ratio is noted as FAR, all on the Y axis; * denotes $p<0.05$.

\subsection{Improvement in Glycated Hemoglobin (HbA1c)}

Although many patients with gastroparesis have co-existing diabetes, few studies have been conducted to explore the effects of GES on the parameters of diabetes. A prospective single-center study on the long-term effects (12 months) of high-frequency/low-energy GES, on symptoms, gastric emptying and metabolic control (HbA1c) in insulin-dependent diabetic subjects with drug-refractory gastroparesis, was conducted on 17 patients. Post-GES, HbA1c values were lowered in all 17 subjects. Initially, all $\mathrm{HbA} 1 \mathrm{c}$ values were above $7.5 \%$; at 6 and 12 months, mean values had significantly decreased from $8.6 \%$ to $6.2 \%$ and $6.5 \%$, respectively. This study, published in 2005 , showed that GES played a role in improving glycohemoglobin, a surrogate for complications from diabetes, in patients with insulin-dependent diabetes [33] (Figure 9). 


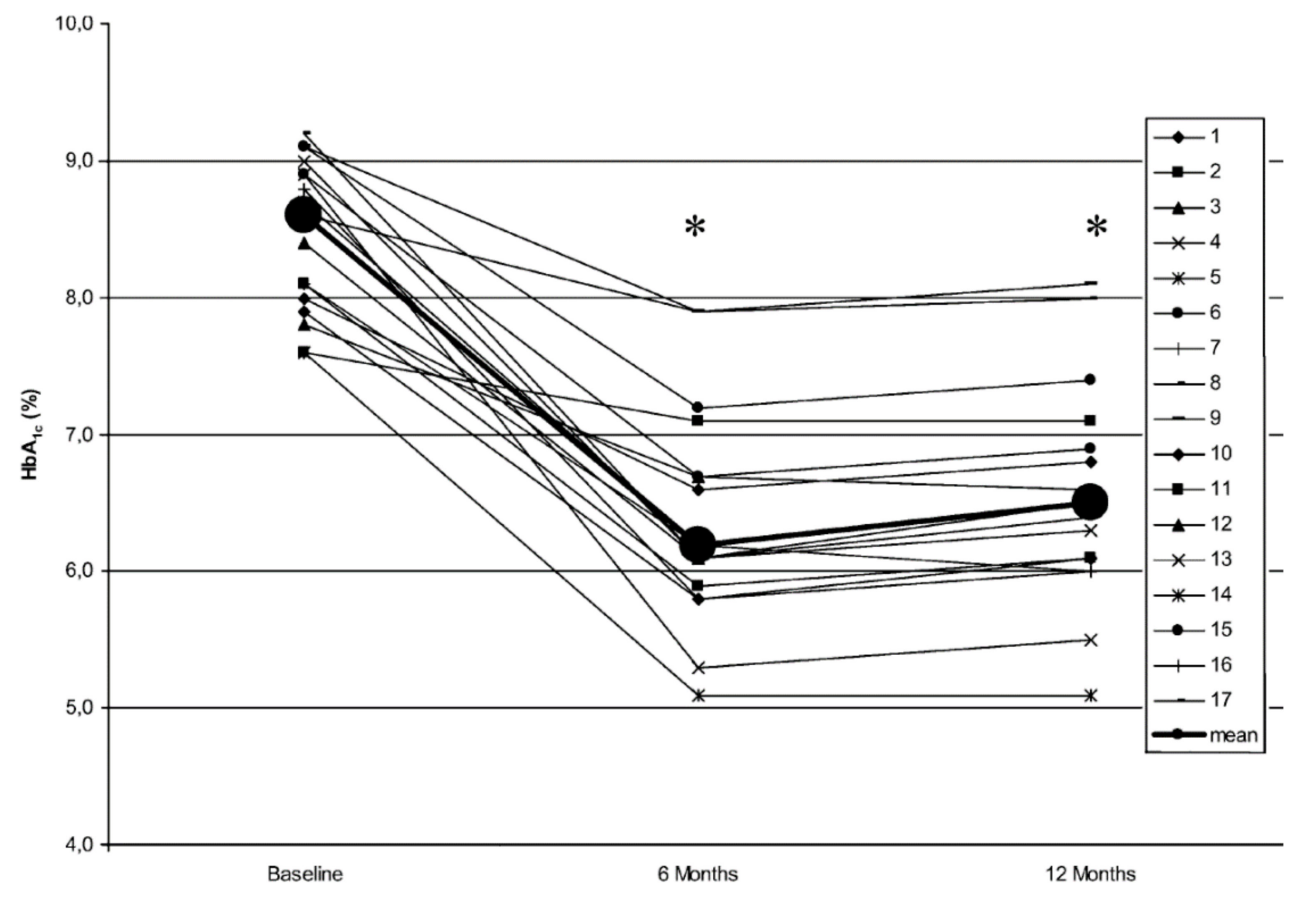

Figure 9. Improvement in A1c. Individual $\mathrm{HbA} 1 \mathrm{c}$ values of all patient at baseline, 6 and 12 months after follow up. In all cases, HbA1c levels were lowered; * denotes $p<0.01$ compared to baseline [33].

\subsection{Effect on Mortality}

A 2005 study [34] examined the survival benefits of GES. This multicenter report was the first to compare long term survival of patients undergoing GES for drug-refractory gastroparesis with historical controls. Out of 123 patients, 19 died, most from primary illnesses. Patient death rates were higher for diabetic patients than for nondiabetic patients, and patient death rates were lower for diabetic patients implanted with GES than for diabetic non-implanted controls. Since the number of diabetic non-implanted controls was small, a survival benefit could not be definitely proven based on this cohort alone. This study, however, emphasized the possible role of GES in improving survival in some patients with diabetic gastroparesis (Figure 10).

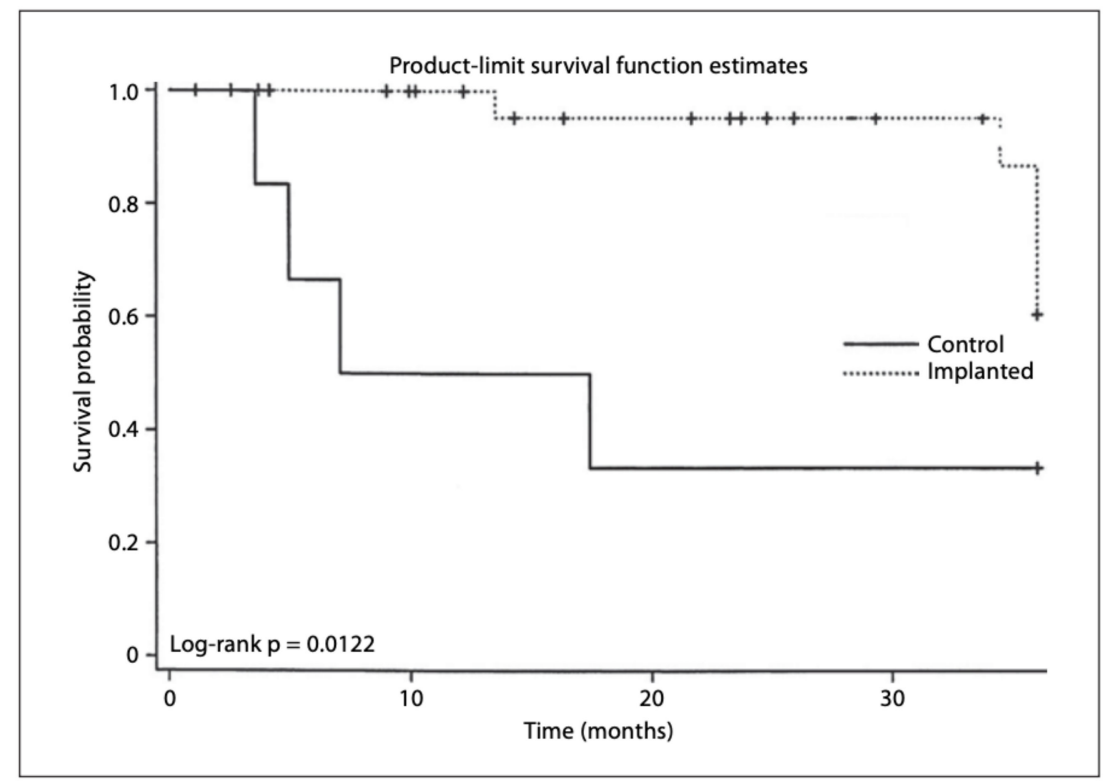

Figure 10. All diabetic implanted versus nonimplanted patients. Survival was lower for diabetic patients not implanted with the GES device [34]. 


\section{Conclusions}

Gastroparetic syndromes have a significant impact on a patient's quality of life and are associated with significant health-related expenditures. Numerous aspects of the pathophysiology of Gp syndromes have been described, while others are being elucidated. In addition to standard therapies, neuromodulation using low and moderate energy with high-frequency gastric electrical stimulation is effective in many patients. We now know that GES exerts its effects through the multiple central and peripheral mechanisms discussed above and has the potential to modify disease mechanisms. Further studies, such as neural changes at the cellular level, may shed light on how GES can provide symptom relief and improve gastric motor function and thus patients' symptoms. Additional work with GES, not discussed here, may demonstrate the role of GES alongside the emerging area of pyloric therapies, for patients with delayed gastric emptying and altered pyloric compliance. Future work on gastroparetic syndromes and their treatment may be better defined and directed in terms of pathophysiologic mechanisms for gastrointestinal neuromodulation.

Supplementary Materials: Supplementary materials can be found at http://www.mdpi.com/2624-5647/2/1/3/s1. References [4,22,28,30] are cited in Supplementary Materials.

Author Contributions: A.S.—concept, data review, writing, final approval. T.L.A.—concept, data review, writing, final approval. All authors have read and agreed to the published version of the manuscript.

Acknowledgments: The authors would like to thank the colleagues and staff at the University of Louisville Gastroenterology Motility Clinic, as well as Hani Rashed for helpful manuscript advice, and Catherine McBride for help with Manuscript submission. Thomas L. Abell is a consultant for Sensa and Nuvaira and is an investigator for Allergan, Alfa-Sigma, Censa, Vanda, Alnylam and ClinDome. He is the GI Section Editor for Med Study, GI Stimulation editor for Neuromodulation, GES editor for Wikistim and a reviewer for UpToDate. Thomas L. Abell is the founder of ADEPT-GI, which holds Intellectual Property (IP) on some of the technologies discussed in this article.

Conflicts of Interest: The authors declare no conflicts of interest.

\section{References}

1. Wang, Y.R.; Fisher, R.S.; Parkman, H.P. Gastroparesis-related hospitalizations in the United States: Trends, characteristics, and outcomes, 1995-2004. Am. J. Gastroenterol. 2008, 103, 313-322. [CrossRef]

2. Jayanthi, N.V.G.; Dexter, S.P.L.; Sarela, A.I.; Leeds Gastroparesis Multi-Disciplinary, T. Gastric electrical stimulation for treatment of clinically severe gastroparesis. J. Minim. Access Surg. 2013, 9, 163-167. [CrossRef] [PubMed]

3. Wendorf, G.; Mehta, M.; Stocker, A.; Smith, J.; Abell, T. Endoscopic aspects of temporary gastric electrical stimulator lead placement in patients with gastroparesis and gastroparesis-like syndromes. VideoGIE 2018, 3, 112. [CrossRef] [PubMed]

4. Abell, T.L.; Kedar, A.; Stocker, A.; Beatty, K.; McElmurray, L.; Hughes, M.; Rashed, H.; Kennedy, W.; Wendelschafer-Crabb, G.; Yang, X.; et al. Gastroparesis syndromes: Response to electrical stimulation. Neurogastroenterol. Motil. 2019, 31, e13534. [CrossRef] [PubMed]

5. Abell, T. Treatment Options for Gastroparesis. Available online: https://oley.org/page/Treat_Gastroparesis (accessed on 30 October 2019).

6. Williams, P.A.; Nikitina, Y.; Kedar, A.; Lahr, C.J.; Helling, T.S.; Abell, T.L. Long-term effects of gastric stimulation on gastric electrical physiology. J. Gastrointest. Surg. 2013, 17, 50-56. [CrossRef] [PubMed]

7. Reichenbach, Z.W.; Stanek, S.; Patel, S.; Ward, S.J.; Malik, Z.; Parkman, H.P.; Schey, R. Botulinum Toxin A Improves Symptoms of Gastroparesis. Dig. Dis. Sci. 2019. [CrossRef]

8. Burlen, J.; Runnels, M.; Mehta, M.; Andersson, S.; Ducrotte, P.; Gourcerol, G.; Lindberg, G.; Fullarton, G.; Abrahamsson, H.; Al-Juburi, A.; et al. Efficacy of Gastric Electrical Stimulation for Gastroparesis: US/European Comparison. Gastroenterol. Res. 2018, 11, 349-354. [CrossRef]

9. Abidi, N.; Starkebaum, W.L.; Abell, T.L. An energy algorithm improves symptoms in some patients with gastroparesis and treated with gastric electrical stimulation. Neurogastroenterol. Motil. 2006, 18, 334-338. [CrossRef] 
10. Qin, C.; Sun, Y.; Chen, J.D.; Foreman, R.D. Gastric electrical s;timulation modulates neuronal activity in nucleus tractus solitarii in rats. Auton. Neurosci. 2005, 119, 1-8. [CrossRef]

11. Snodgrass, P.; Sandoval, H.; Calhoun, V.D.; Ramos-Duran, L.; Song, G.; Sun, Y.; Alvarado, B.; Bashashati, M.; Sarosiek, I.; McCallum, R.W. Central Nervous System Mechanisms of Nausea in Gastroparesis: An fMRI-Based Case-Control Study. Dig. Dis. Sci. 2019. [CrossRef]

12. McCallum, R.W.; Dusing, R.W.; Sarosiek, I.; Cocjin, J.; Forster, J.; Lin, Z. Mechanisms of symptomatic improvement after gastric electrical stimulation in gastroparetic patients. Neurogastroenterol. Motil. 2010. [CrossRef] [PubMed]

13. Familoni, B.O.; Abell, T.L.; Bhaskar, S.K.; Voeller, G.R.; Blair, S.R. Gastric electrical stimulation has an immediate antiemetic effect in patients with gastroparesis. IEEE Transact. Biomed. Eng. 2006, 53, 1038-1046. [CrossRef] [PubMed]

14. Abell, T.; McCallum, R.; Hocking, M.; Koch, K.; Abrahamsson, H.; Leblanc, I.; Lindberg, G.; Konturek, J.; Nowak, T.; Quigley, E.M.; et al. Gastric electrical stimulation for medically refractory gastroparesis. Gastroenterology 2003, 125, 421-428. [CrossRef]

15. Abell, T.L.; Van Cutsem, E.; Abrahamsson, H.; Huizinga, J.D.; Konturek, J.W.; Galmiche, J.P.; Voeller, G.; Filez, L.; Everts, B.; Waterfall, W.E.; et al. Gastric electrical stimulation in intractable symptomatic gastroparesis. Digestion 2002, 66, 204-212. [CrossRef]

16. Abell, T.L.; Johnson, W.D.; Kedar, A.; Runnels, J.M.; Thompson, J.; Weeks, E.S.; Minocha, A.; Griswold, M.E. A double-masked, randomized, placebo-controlled trial of temporary endoscopic mucosal gastric electrical stimulation for gastroparesis. Gastrointest. Endosc. 2011, 74, 496-503. [CrossRef]

17. Gourcerol, G.; Ouelaa, W.; Huet, E.; Leroi, A.M.; Ducrotte, P. Gastric electrical stimulation increases the discomfort threshold to gastric distension. Eur. J. Gastroenterol. Hepatol. 2013, 25, 213-217. [CrossRef]

18. Shine, A.; Ahmed, S.; McElmurray, L.; Stocker, A.; Pinkston, C.; Abell, T. 116 - Electrogastography At Baseline and Response to Temporary Gastric Electrical Stimulation - a Comparison of Cutaneous with Mucosal Recordings. Gastroenterology 2019, 156, S30-S31. [CrossRef]

19. Pasricha, P.J.; Mehta, K.R.; May, K.P.; Nguyen, L.A.B.; Abell, T.L.; Parkman, H.P.; Hasler, W.L.; Koch, K.L.; Calles, J.; McCallum, R. Characterization of the inflammatory response in idiopathic gastroparesis reveals a role for macrophage derived factors. Gastroenterology 2011, 140, S-709. [CrossRef]

20. Gallas, S.; Sinno, M.H.; Boukhettala, N.; Coeffier, M.; Dourmap, N.; Gourcerol, G.; Ducrotte, P.; Dechelotte, P.; Leroi, A.M.; Fetissov, S.O. Gastric electrical stimulation increases ghrelin production and inhibits catecholaminergic brainstem neurons in rats. Eur. J. Neurosci. 2011, 33, 276-284. [CrossRef]

21. Liu, S.; Tang, M.; Tao, S.; Chen, J.D. Central expressions of ghrelin and cholecystokinin in rats with gastric electrical stimulation. Obes. Surg. 2008, 18, 109-114. [CrossRef]

22. Abell, T.; Lou, J.; Tabbaa, M.; Batista, O.; Malinowski, S.; Al-Juburi, A. Gastric electrical stimulation for gastroparesis improves nutritional parameters at short, intermediate, and long-term follow-up. JPEN. J. Parent. Ent. Nutrit. 2003, 27, 277-281. [CrossRef] [PubMed]

23. Stocker, A.; Abell, T.L.; Rashed, H.; Kedar, A.; Boatright, B.; Chen, J. Autonomic Evaluation of Patients With Gastroparesis and Neurostimulation: Comparisons of Direct/Systemic and Indirect/Cardiac Measures. Gastroenterol. Res. 2016, 9, 10-16. [CrossRef] [PubMed]

24. Chowdhury, R.S.; Forsmark, C.E.; Davis, R.H.; Toskes, P.P.; Verne, G.N. Prevalence of Gastroparesis in Patients with Small Duct Chronic Pancreatitis. Pancreas 2003, 26, 235-238. [CrossRef] [PubMed]

25. Luo, J.; Al-Juburi, A.; Rashed, H.; O’Dorisio, T.; Marchal, B.; Starkebaum, W.; Abell, T.L. Gastric electrical stimulation is associated with improvement in pancreatic exocrine function in humans. Pancreas 2004, 29, e41-e44. [CrossRef]

26. McCallum, R.W.; Sarosiek, I.; Parkman, H.P.; Snape, W.; Brody, F.; Wo, J.; Nowak, T. Gastric electrical stimulation with Enterra therapy improves symptoms of idiopathic gastroparesis. Neurogastroenterol. Motil. 2013. [CrossRef]

27. Li, H.; Chen, Y.; Liu, S.; Hou, X.-H. Long-pulse gastric electrical stimulation protects interstitial cells of Cajal in diabetic rats via IGF-1 signaling pathway. World J. Gastroenterol. 2016, 22, 5353-5363. [CrossRef]

28. Omer, E.; Kedar, A.; Nagarajarao, H.S.; Nikitina, Y.; Vedanarayanan, V.; Subramony, C.; Lahr, C.J.; Abell, T.L. Cajal Cell Counts are Important Predictors of Outcomes in Drug Refractory Gastroparesis Patients With Neurostimulation. J. Clin. Gastroenterol. 2019, 53, 366-372. [CrossRef] 
29. Cutts, T.F.; Luo, J.; Starkebaum, W.; Rashed, H.; Abell, T.L. Is gastric electrical stimulation superior to standard pharmacologic therapy in improving GI symptoms, healthcare resources, and long-term health care benefits? Neurogastroenterol. Motil. 2005, 17, 35-43. [CrossRef]

30. Lahr, C.J.; Griffith, J.; Subramony, C.; Halley, L.; Adams, K.; Paine, E.R.; Schmieg, R.; Islam, S.; Salameh, J.; Spree, D.; et al. Gastric electrical stimulation for abdominal pain in patients with symptoms of gastroparesis. Am. Surg. 2013, 79, 457-464.

31. Agrawal, A.; Francis, S.L.; Deveneau, N.E.; Jain, S.; Abrasley, C.; McNeese, J.T.; Kothari, S.T.; Lahr, C.J.; Abell, T.L. Gastric Electrical Stimulation and Sacral Electrical Stimulation: A Long-Term Follow-Up Study of Dual-Device Treatment. Dig. Dis. Sci. 2016, 61, 176-180. [CrossRef]

32. McKenzie, P.; Stocker, A.; Du, P.; Lahr, C.; Cheng, L.K.; McElmurray, L.; Kedar, A.; Boatright, B.; Hassan, H.; Hughes, M.; et al. The Effect of Gastric Electrical Stimulation on Small Bowel Motility in Patients With Gastroparesis and Concomitant Pancreatic and Small Bowel Dysfunction: From Animal Model to Human Application. Neuromodulation 2019, 22, 723-729. [CrossRef] [PubMed]

33. Van der Voort, I.R.; Becker, J.C.; Dietl, K.H.; Konturek, J.W.; Domschke, W.; Pohle, T. Gastric electrical stimulation results in improved metabolic control in diabetic patients suffering from gastroparesis. Exper. Clin. Endocrinol. Diab. 2005, 113, 38-42. [CrossRef] [PubMed]

34. Anand, C.; Al-Juburi, A.; Familoni, B.; Rashed, H.; Cutts, T.; Abidi, N.; Johnson, W.D.; Minocha, A.; Abell, T.L. Gastric electrical stimulation is safe and effective: A long-term study in patients with drug-refractory gastroparesis in three regional centers. Digestion 2007, 75, 83-89. [CrossRef] [PubMed]

(C) 2020 by the authors. Licensee MDPI, Basel, Switzerland. This article is an open access article distributed under the terms and conditions of the Creative Commons Attribution (CC BY) license (http://creativecommons.org/licenses/by/4.0/). 